\title{
Two circular-rotational eigenmodes and their giant resonance asymmetry in vortex gyrotropic motions in soft magnetic nanodots
}

\author{
Ki-Suk Lee and Sang-Koog Kim* \\ Research Center for Spin Dynamics and Spin-Wave Devices, Seoul National University, Seoul 151-744, Republic of Korea \\ and Nanospinics Laboratory, Department of Materials Science and Engineering, College of Engineering, Seoul National \\ University, Seoul 151-744, Republic of Korea
}

(Received 9 April 2008; published 3 July 2008)

\begin{abstract}
We found, by micromagnetic numerical and analytical calculations, that the clockwise $(\mathrm{CW})$ and counterclockwise (CCW) circular-rotational motions of a magnetic vortex core in a soft magnetic circular nanodot are the elementary eigenmodes existing in the gyrotropic motion with respect to the corresponding $\mathrm{CW}$ and $\mathrm{CCW}$ circular-rotational-field eigenbasis. The oppositely rotating eigenmodes show a giant asymmetric resonance behavior, i.e., for the up-core orientation the $\mathrm{CCW}$ eigenmode shows a strong resonance at the field frequency equal to the vortex eigenfrequency, but the other $\mathrm{CW}$ eigenmode shows nonresonance. This asymmetric resonace effect is reversed by changing the vortex polarization. The orbital radius amplitudes and phases of the two circular eigenmodes vary with the polarization and chirality of the given vortex state as well as the field frequency. The overall linear-regime steady-state vortex gyrotropic motions driven by arbitrary polarized oscillating in-plane magnetic field in the linear regime can be perfectly understood according to the superposition of the two circular eigenmodes.
\end{abstract}

DOI: 10.1103/PhysRevB.78.014405

PACS number(s): 75.40.Gb, 75.40.Mg, 75.75.+a

\section{INTRODUCTION}

The magnetic vortex consists of the in-plane curling magnetizations (M's) and the out-of-plane M's at the center region, the so-called vortex core (VC) ${ }^{1-3}$ which is known to be a ground state in soft magnetic elements of micron size or smaller. When, in such a confined system, magnetic fields (or currents) with harmonic oscillations or pulses are applied to the vortex, its $\mathrm{VC}$ rotates around its equilbrium position at a characteristic eigenfrequency, $\omega_{D} / 2 \pi$, typically, of several hundred megahertz. ${ }^{4-10}$ The responsible force is the gyroforce exerting on the $\mathrm{VC}$, which is in balance with the restoring force due mainly to the long-range dipole-dopole interaction dominating in a confined magnetic element. ${ }^{5}$ Such vortex excitation is known to be the translation mode or gyrotropic motion of a $\mathrm{VC}$ in the dot plane, and the rotation sense of such gyrotropic motion is determined by the polarization $p$ of a given vortex, which is represented by the VC $\mathbf{M}$ orientation [ $p=+1(-1)$ for up (down)-core orientation]. If the angular frequency $\omega_{\mathbf{H}}$ of an oscillating field (or current) is close to the $\omega_{D},{ }^{5}$ the VC motion is resonantly excited. ${ }^{8-12}$ Recently, this resonantly excited VC gyrotropic motions under harmonic oscillating field, ${ }^{8,9}$ ac current, ${ }^{10}$ or both of them ${ }^{11}$ have been intensively studied. Moreover, the resonant VC motion has attracted much attention on account of its related ultrafast VC switching applicable to information storage. ${ }^{13-18}$ In addition, the variation of the circular and elliptical shapes of the orbital trajectories of the on- and offresonance VC motions driven by linearly polarized oscillating magnetic fields was observed. ${ }^{7,8}$ However, the underlying physics has not been clearly understood since the true eigenmodes of the vortex gyrotropic motions remain incompletely understood. In this paper, having considered the results of the present theoretical and numerical simulation studies, we posit that these VC motions can be clearly understood by considering them to be the superposition of counterclockwise $(\mathrm{CCW})$ and clockwise $(\mathrm{CW})$ circular-rotational eigenmodes and also by considering their aysmmetric resonance effect. The orbital-trajectory radius ampltidues and phases of the oppositely rotating eigenmodes' VC motions are presented as a fucntion of the frequecy of oscillating fields according to the different vortex polarizations and chiralities.

\section{MICROMAGNETIC SIMULATIONS}

In the present study, we employed micromagnetic numerical simulations of vortex $\mathbf{M}$ dynamics by using the OOMMF code $^{19}$ that utilizes the Landau-Lifshitz-Gilbert equation of motion $\partial \mathbf{M} / \partial t=-\gamma\left(\mathbf{M} \times \mathbf{H}_{e f f}\right)+\alpha /|\mathbf{M}|(\mathbf{M} \times \partial \mathbf{M} / \partial t)($ Ref. 20) with the phenomenological damping constant $\alpha$, the gyromagnetic ratio $\gamma$, and the effective field $\mathbf{H}_{\text {eff. }}$. Also, we carried out analytical calculations of the linear-regime VC motions, ${ }^{8}$ which are based on a linearized Thiele's equation of motion. ${ }^{21}$ As a model system, we chose a Permalloy (Py) nanodot of $2 R=300 \mathrm{~nm}$ diameter and $L=10 \mathrm{~nm}$ thickness [Fig. 1(a)]. For the given Py material and circular dot geometry, a single magnetic vortex is present with either $p=+1$ or -1 and with either $C=+1$ or -1 , where $C=+1(-1)$ is the chirality, which is represented by the $\mathrm{CCW}(\mathrm{CW})$ in-plane M's around the VC. The vortex eigenfrequency and static annihilation field (Ref. 22) were estimated to be $\omega_{D} / 2 \pi=330 \mathrm{MHz}$ and $H_{A}=500 \mathrm{Oe}$, respectively. We considered the application of either linearly polarized oscillating magnetic fields applied along the $y$ axis, $\mathbf{H}_{\text {lin }}=H_{0} \sin \left(\omega_{\mathbf{H}} t\right) \hat{\mathbf{y}}$, or circularly polarized oscillating fields of either $\mathrm{CCW}$ or $\mathrm{CW}$ rotation sense in the dot plane, such that $\mathbf{H}_{\mathrm{CCW}}=\frac{1}{2}\left[H_{0} \cos \left(\omega_{\mathbf{H}} t\right) \hat{\mathbf{x}}+H_{0} \sin \left(\omega_{\mathbf{H}} t\right) \hat{\mathbf{y}}\right]$ and $\mathbf{H}_{\mathrm{CW}}=\frac{1}{2}\left[-H_{0} \cos \left(\omega_{\mathbf{H}} t\right) \hat{\mathbf{x}}+H_{0} \sin \left(\omega_{\mathbf{H}} t\right) \hat{\mathbf{y}}\right]$ with the oscillating field amplitude $H_{0}$. We used relatively low field amplitudes, $H_{0} / H_{A}=0.1$ and 0.2 , in investigations of only linear-regime vortex gyrotropic motions, so as to exclude the nonlinear effect $^{8,9}$ driven by high-strength fields. We also chose a frequency range from $\omega_{\mathbf{H}} / 2 \pi=100$ to $825 \mathrm{MHz}$, including $\omega_{D} / 2 \pi=330 \mathrm{MHz}$ of interest, in order to investigate both the 

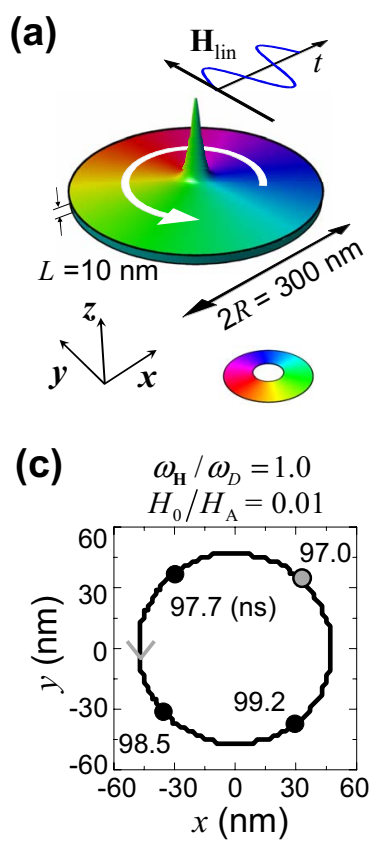

(d)

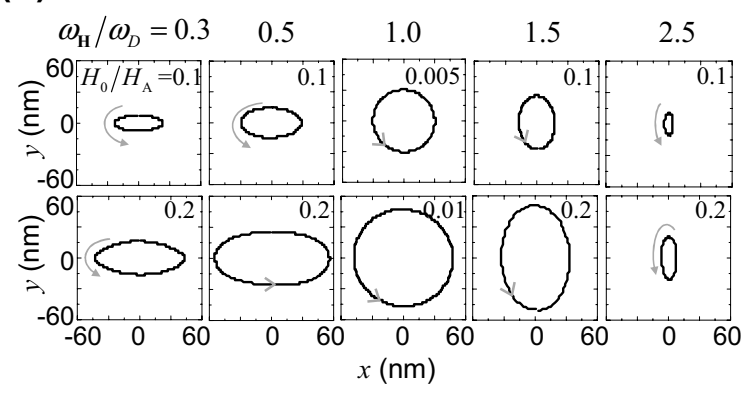

FIG. 1. (Color online) (a) Geometry and dimension of the model Py nanodot with a vortex-state $\mathbf{M}$ distribution with $p=+1$ and $C$ $=+1$ at equilibrium under no field. (b) Perspective view of the local $\mathbf{M}$ distributions at the indicated times and (c) the circular orbital trajectory of the VC motion in the steady state, which is driven by $\mathbf{H}_{\text {lin }}$. The color and height display the local in-plane $\mathbf{M}$ orientation, as indicated by the color wheel, as well as the out-of-plane $\mathbf{M}$ components, respectively. The dots in (c) indicate the VC positions at the indicated times. (d) Orbital trajectories of the steady-state VC motions ( $t>90 \mathrm{~ns}$ ) in response to the $\mathbf{H}_{\text {lin }}$ with different $\omega_{\mathbf{H}}$ values as noted for $H_{0} / H_{A}=0.1$ and 0.2 .

on- and off-resonance vortex gyrotropic motions.

First, we have to note that the orbital trajectory of the $\mathbf{H}_{\text {lin }}$-driven steady-state VC motion for the resonance case $\omega_{\mathbf{H}}=\omega_{D}$ is exactly circular in shape and relatively large in radius amplitude, even for a very weak field strength, e.g., $H_{0} / H_{A}=0.01$ (Ref. 23) [see Figs. 1(b) and 1(c) and Supplementary Movie 1 (Ref. 24)]. For $p=+1$, the VC motion is $\mathrm{CCW}$ and for $p=-1, \mathrm{CW}$. In the case of off-resonance $\left(\omega_{\mathbf{H}}\right.$ $\left.\neq \omega_{D}\right)$ motion, the orbits, however, become elongated along the axis perpendicular to and parallel with the $\mathbf{H}_{\text {lin }}$ axis for $\omega_{\mathbf{H}}<\omega_{D}$ and $\omega_{\mathbf{H}}>\omega_{D}$, respectively, and the degree of their elongations increases with the increasing magnitude of $\mid \omega_{\mathbf{H}}$ $-\omega_{D} \mid\left[\right.$ Fig. 1(d)]. ${ }^{8,11}$ Although such behaviors have been reported from previous numerical and analytical studies, ${ }^{7,8,11}$ the underlying physics has not been understood yet. (a)
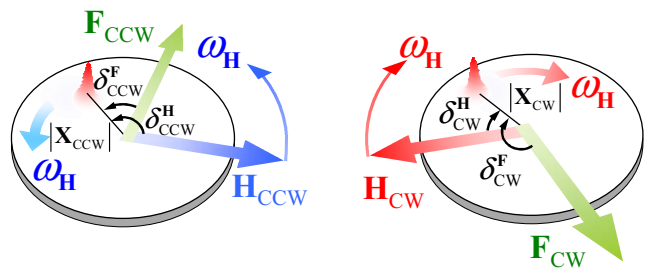

(b)

(c)
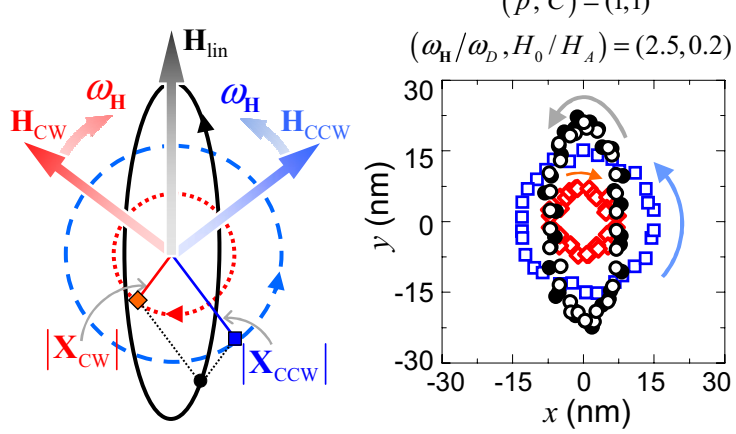

FIG. 2. (Color online) (a) Graphical illustrations of the CCW and $\mathrm{CW}$ circular eigenmodes and the corresponding $\mathbf{H}_{\mathrm{CCW}}, \mathbf{H}_{\mathrm{CW}}$, $\mathbf{F}_{\mathrm{CCW}}$, and $\mathbf{F}_{\mathrm{CW}}$, as well as the definitions of the orbital-radius amplitude $\left|\mathbf{X}_{\mathrm{CCW}, \mathrm{CW}}\right|$ and phase $\delta_{\mathrm{CCW}, \mathrm{CW}}^{\mathrm{H}}$ of the linear-regime steadystate circular VC motions. (b) Graphical illustration of the superposition of the CCW and CW eigenmodes, yielding an overall elliptical orbit. The black-colored ellipse (solid line) indicates the resultant superposition of the individual $\mathrm{CCW}$ [blue-colored (dashed) line] and CW [red-colored (dotted) line] eigenmodes, which is equivalent to the elliptical $\mathrm{VC}$ motion driven by the $\mathbf{H}_{\text {lin }}$ (black arrow). The $\mathrm{VC}$ positions of the $\mathrm{CCW}$ and $\mathrm{CW}$ eigenmotions at a certain time are indicated by the blue-colored square and redcolored diamond, respectively, and their vector sum is indicated by the black-colored dot on the ellipse. (c) Micromagnetic simulation results on the VC trajectories of the CCW (blue open squares) and $\mathrm{CW}$ (red open diamonds) eigenmotions driven by the individual $\mathbf{H}_{\mathrm{CCW}}$ and $\mathbf{H}_{\mathrm{CW}}$, respectively, as well as the VC trajectory (black open circles) driven by the $\mathbf{H}_{\text {lin }}$. The elliptical orbit (black solid circles) results from the superposition of the individual $\mathrm{CCW}$ and $\mathrm{CW}$ eigenmotions.

For clear understanding, it is thus necessary to find out the elementary eigenmodes of the gyrotropic motions. The application of the $\mathbf{H}_{\text {lin }}$ is equivalent to the application of both the pure circular fields of $\mathbf{H}_{\mathrm{CCW}}$ and $\mathbf{H}_{\mathrm{CW}}$ simultaneously, with the same $H_{0}$ and with equal $\omega_{\mathbf{H}}$ [Fig. 2(a)], because $\mathbf{H}_{\text {lin }}$ can, in principle, be decomposed into the $\mathbf{H}_{\mathrm{CCW}}$ and $\mathbf{H}_{\mathrm{CW}}$ components. ${ }^{15}$ The relative phase between $\mathbf{H}_{\mathrm{CCW}}$ and $\mathbf{H}_{\mathrm{CW}}$ determines the axis of the linearly polarized oscillating magnetic field. Thus, the observed circular or elliptical shape of the orbital trajectories [Fig. 1(d)] can be interpreted according to the superposition of the $\mathrm{CCW}$ and $\mathrm{CW}$ circular eigenmotions in circular dots with respect to the $\mathbf{H}_{\mathrm{CCW}}$ and $\mathbf{H}_{\mathrm{CW}}$ eigenbasis, as seen in Fig. 2(b) [see Supplementary Movie 2 (Ref. 24)]. To verify this by micromagnetic simulations, in Fig. 2(c), we plotted the individual orbital trajectories of the VC motions under the individuals of the $\mathbf{H}_{\mathrm{CCW}}$ and $\mathbf{H}_{\mathrm{CW}}$, as well as the $\mathbf{H}_{\text {lin }}$ for a specific case of $\omega_{\mathbf{H}} / \omega_{D}=2.5, H_{0} / H_{A}$ $=0.2$, and $(p, C)=(+1,+1)$. The elliptical orbital trajectory by $\mathbf{H}_{\text {lin }}$ is in excellent agreement with that obtained by the 
superposition of the $\mathrm{CCW}$ and $\mathrm{CW}$ circular-rotational motions.

\section{ANALYTICAL CALCULATIONS}

\section{Two circular-rotational eigenmodes}

In order to theoretically verify and understand such dynamic responses of a vortex to any polarized oscillating field, now we introduce a useful quantity of the dynamic susceptibility tensor $\hat{\chi}_{\mathbf{X}}$ defined by $\mathbf{X}=\hat{\chi}_{\mathbf{X}} \mathbf{H} .{ }^{12}$ For convenience, first let us define the orbital radius $\left|\mathbf{X}_{\mathrm{CCW}, \mathrm{CW}}\right|$ and phase $\delta_{\mathrm{CCW}, \mathrm{CW}}^{\mathbf{H}}$ of the VC position $\mathbf{X}$ in the dot $(x-y)$ plane for the CCW and CW circular motions [Figs. 2(a) and 2(b)]. Here, we exclude nonsteady transient-state motions that have yet to reach the steady state, as well as the nonlinear effect. ${ }^{8,9}$ To analytically calculate $\left|\mathbf{X}_{\mathrm{CCW}, \mathrm{CW}}\right|$ and $\delta_{\mathrm{CCW}, \mathrm{CW}}^{\mathbf{H}}$, we employed the linearized Thiele's equation ${ }^{21}$ of motion, $-\mathbf{G} \times \dot{\mathbf{X}}-\hat{D} \dot{\mathbf{X}}$ $+\partial W(\mathbf{X}, t) / \partial \mathbf{X}=0$, with the gyrovector $\mathbf{G}=-G \hat{\mathbf{z}}$, and the damping tensor $\hat{D}=D \hat{I}$ with the identity matrix $\hat{I}$ and the damping constant $D .^{5,12}$ The potential energy function is given by $W(\mathbf{X}, t)=W(0)+\kappa|\mathbf{X}|^{2} / 2+W_{\mathbf{H}}$. The first term $W(0)$ is the potential energy for a $\mathrm{VC}$ at its initial position $\mathbf{X}=0$, and the second term is dominated by the exchange and magnetostatic energies for the VC shift from $\mathbf{X}=0$ and for the given stiffness coefficient $\kappa$. The last one, $W_{\mathbf{H}}=-\mu(\hat{\mathbf{z}}$ $\times \mathbf{H}) \cdot \mathbf{X}$, is the Zeeman energy term due to a driving force, where $\mu=\pi R L M_{s} \xi C$, with $\xi=2 / 3$ for the "side-charge-free" model. $5,8,12$ For any polarized oscillating field $\mathbf{H}$ $=\mathbf{H}_{0} \exp \left(-i \omega_{\mathbf{H}} t\right)$, i.e., linearly, circularly polarized oscillating field, or their mixed polarizations, the general solution is written as $\mathbf{X}=\mathbf{X}^{\text {trans }}+\mathbf{X}^{\text {steady }}{ }^{11}$ where the terms $\mathbf{X}^{\text {trans }}$ and $\mathbf{X}^{\text {steady }}$ correspond to the VC motions in the transient and field-driven steady states, respectively, given by $\mathbf{X}^{\text {trans }}=$ $-\mathbf{X}_{0} \exp \left(-i \omega_{D} t\right) \exp \left(D \omega_{D} t /|G|\right) \quad$ with $\omega_{D}=\kappa|G| /\left(G^{2}+D^{2}\right)$ (Ref. 17) and $\mathbf{X}^{\text {steady }}=\mathbf{X}_{0} \exp \left(-i \omega_{\mathbf{H}} t\right)$. For $t \gg\left|G /\left(D \omega_{D}\right)\right|(t$ $\gg 23 \mathrm{~ns}$ in this case), the $\mathrm{VC}$ motions can be represented by $\mathbf{X} \simeq \mathbf{X}^{\text {steady }}=\mathbf{X}_{0} \exp \left(-i \omega_{\mathbf{H}} t\right)$. For the linear polarization basis, the susceptibility tensor is $\mathbf{X}_{0, L}=\hat{\chi} \mathbf{x}_{, L} \mathbf{H}_{0, L}$ with $\mathbf{X}_{0, L}=X_{0 x} \hat{\mathbf{x}}$ $+X_{0 y} \hat{\mathbf{y}}$ and $\mathbf{H}_{0, L}=H_{0 x} \hat{\mathbf{x}}+H_{0 y} \hat{\mathbf{y}}$, where

$$
\begin{aligned}
\hat{\chi}_{X, L}\left(\omega_{\mathbf{H}}\right) & =\left[\begin{array}{ll}
\chi_{x x} & \chi_{x y} \\
\chi_{y x} & \chi_{y y}
\end{array}\right] \\
& =\frac{\mu}{\left(i \omega_{\mathbf{H}} D+\kappa\right)^{2}-\left(\omega_{\mathbf{H}} G\right)^{2}}\left[\begin{array}{cc}
-i \omega_{\mathbf{H}} G & -i \omega_{\mathbf{H}} D-\kappa \\
i \omega_{\mathbf{H}} D+\kappa & -i \omega_{\mathbf{H}} G
\end{array}\right] .
\end{aligned}
$$

By the diagonalization of $\hat{\chi}_{X}, \mathbf{X}_{0, L}=\hat{\chi}_{\mathbf{X}, L} \mathbf{H}_{0, L}$ becomes $\mathbf{X}_{0 \text {,cir }}$ $=\hat{\chi} \mathbf{x}$, cir $\mathbf{H}_{0, \text { cir }}$ with respect to the circular polarization eigenbasis, where $\mathbf{H}_{0, \mathrm{cir}}=H_{0, \mathrm{CCW}} \hat{\mathbf{e}}_{\mathrm{CCW}}+H_{0, \mathrm{CW}} \hat{\mathbf{e}}_{\mathrm{CW}}$ and $\mathbf{X}_{0, \mathrm{cir}}$ $=X_{0, \mathrm{CCW}} \hat{\mathbf{e}}_{\mathrm{CCW}}+X_{0, \mathrm{CW}} \hat{\mathbf{e}}_{\mathrm{CW}}$ with the circular eigenvectors of $\hat{\mathbf{e}}_{\mathrm{CCW}}=\frac{1}{\sqrt{2}}(\hat{\mathbf{x}}+i \hat{\mathbf{y}})$ and $\hat{\mathbf{e}}_{\mathrm{CW}}=\frac{1}{\sqrt{2}}(\hat{\mathbf{x}}-i \hat{\mathbf{y}}) \cdot{ }^{25} \mathbf{X}_{0, \mathrm{cir}}=\hat{\chi} \mathbf{X}$, cir $\mathbf{H}_{0, \mathrm{cir}}$ can also be rewritten, in matrix form, as

$$
\left(\begin{array}{c}
X_{0, \mathrm{CCW}} \\
X_{0, \mathrm{CW}}
\end{array}\right)=\left(\begin{array}{cc}
\chi_{\mathrm{CCW}} & 0 \\
0 & \chi_{\mathrm{CW}}
\end{array}\right)\left(\begin{array}{c}
H_{0, \mathrm{CCW}} \\
H_{0, \mathrm{CW}}
\end{array}\right),
$$

where $\chi_{\mathrm{CCW}}\left(\omega_{\mathbf{H}}\right)=i \mu /\left[\omega_{\mathbf{H}} G-\left(i \omega_{\mathbf{H}} D+\kappa\right)\right]$ and $\chi_{\mathrm{CW}}\left(\omega_{\mathbf{H}}\right)$ $=i \mu /\left[\omega_{\mathbf{H}} G+\left(i \omega_{\mathbf{H}} D+\kappa\right)\right]$. The term $\chi_{\mathrm{CCW}, \mathrm{CW}}$ can be sepa-
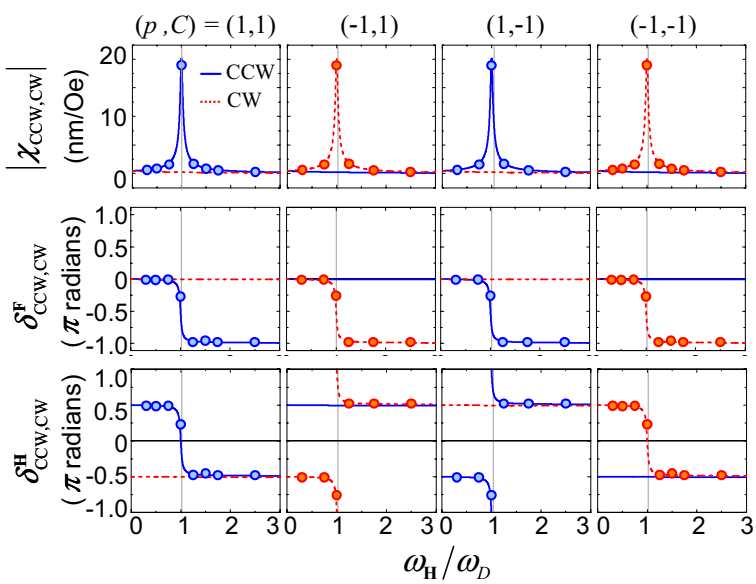

FIG. 3. (Color online) Numerical calculations of the analytical equations (solid and dashed lines) of $\left|\chi_{\mathrm{CCW}, \mathrm{CW}}\left(\omega_{\mathbf{H}}\right)\right|, \delta_{\mathrm{CCW}, \mathrm{CW}}^{\mathbf{F}}\left(\omega_{\mathbf{H}}\right)$, and $\delta_{\mathrm{CCW}, \mathrm{CW}}^{\mathbf{H}}\left(\omega_{\mathbf{H}}\right)$ compared to micromagnetic simulations (shaded circles) for the $\mathrm{CCW}$ and $\mathrm{CW}$ eigenmodes in response to $\mathbf{H}_{\mathrm{CCW}}$ [blue (solid)] and $\mathbf{H}_{\mathrm{CW}}$ [red (dashed)], respectively, for the given polarization and chirality $(p, C)$, as noted.

rated into the magnitude $\left|\chi_{\mathrm{CCW}, \mathrm{CW}}\right|$ and phase $\delta_{\mathrm{CCW}, \mathrm{CW}}^{\mathrm{H}}$ by the relation of $\chi_{\mathrm{CCW}, \mathrm{CW}}=\left|\chi_{\mathrm{CCW}, \mathrm{CW}}\right| e^{-i \delta_{\mathrm{CCW}, \mathrm{CW}}^{\mathrm{H}} \text {, where }}$

$$
\begin{aligned}
& \left|\chi_{\mathrm{CCW}}\right|=|\mu| / \sqrt{\left(G^{2}+D^{2}\right)\left(\omega_{\mathbf{H}}-p \omega_{D}\right)^{2}+\kappa^{2} D^{2} /\left(G^{2}+D^{2}\right)}, \\
& \left|\chi_{\mathrm{CW}}\right|=|\mu| / \sqrt{\left(G^{2}+D^{2}\right)\left(\omega_{\mathbf{H}}+p \omega_{D}\right)^{2}+\kappa^{2} D^{2} /\left(G^{2}+D^{2}\right)},
\end{aligned}
$$

and

$$
\begin{gathered}
\delta_{\mathrm{CCW}}^{\mathbf{H}}=-\tan ^{-1}\left[\left(\kappa-p \omega_{\mathbf{H}}|G|\right) /\left(\omega_{\mathbf{H}} D\right)\right]+\frac{\pi}{2}(1-C), \\
\delta_{\mathrm{CW}}^{\mathbf{H}}=-\tan ^{-1}\left[\left(\kappa+p \omega_{\mathbf{H}}|G|\right) /\left(\omega_{\mathbf{H}} D\right)\right]+\frac{\pi}{2}(1+C) .
\end{gathered}
$$

Both $\left|\chi_{\mathrm{CCW}, \mathrm{CW}}\right|$ and $\delta_{\mathrm{CCW}, \mathrm{CW}}^{\mathbf{H}}$ are functions of $\omega_{\mathbf{H}}$, indicating their $\omega_{\mathbf{H}}$ variations. $\left|\chi_{\mathrm{CCW}, \mathrm{CW}}\right|$ also depend on the sign of $p$ but independently of the sign of $C$, whereas $\delta_{\mathrm{CCW}, \mathrm{CW}}^{\mathbf{H}}$ depend on the sign of both $p$ and $C$.

Next, the numerical calculations of the analytically derived equations of $\left|\chi_{\mathrm{CCW}, \mathrm{CW}}\right|$ and $\delta_{\mathrm{CCW}, \mathrm{CW}}^{\mathrm{H}}$ were plotted versus $\omega_{\mathbf{H}} / \omega_{D}$ for four different cases of $(p, C)$ in Fig. 3, which are in excellent agreements with the simulation results (circle symbols) obtained from the relation of $\left|\chi_{\mathrm{CCW}, \mathrm{CW}}\right|$ $=\left|\mathbf{X}_{\mathrm{CCW}, \mathrm{CW}}\right| /\left|\mathbf{H}_{\mathrm{CCW}, \mathrm{CW}}\right|$. There exist strong resonances for both $\left|\chi_{\mathrm{CCW}, \mathrm{CW}}\right|$ and $\delta_{\mathrm{CCW}, \mathrm{CW}}$ at $\omega_{\mathbf{H}} / \omega_{D}=1$ and the resonance effects are asymmetric between the $\mathrm{CCW}$ and $\mathrm{CW}$ circular motions. Only one, either the $\mathrm{CCW}$ or $\mathrm{CW}$ motion, shows a resonance behavior, the other showing nonresonance. This asymmetric resonance is caused by the gyroforce $(\mathbf{G} \times \dot{\mathbf{X}})$, which is essential for vortex gyrotropic motion. The presence of the gyroforce leads to a broken time-reversal symmetry, in turn, yielding a splitting of the degeneracy of the $\mathrm{CCW}$ and $\mathrm{CW}$ eigenmodes. Therefore, the vortex gyrotropic motion shows such asymmetric resonance, responding differently to the orthogonal $\mathrm{CCW}$ and $\mathrm{CW}$ circular fields. The asymmetric resonance effect is reversed by changing from $p=+1$ to -1 , 
i.e., the mode showing the resonance is switched by $p$. This can also be simply confirmed by the on-resonance case equations, $\left|\chi_{\mathrm{CCW}}\left(\omega_{D}\right)\right|=(|\mu| / \kappa) \sqrt{\left(G^{2}+D^{2}\right) / D^{2}}$ and $\left|\chi_{\mathrm{CW}}\left(\omega_{D}\right)\right|=(|\mu| / \kappa) \sqrt{\left(G^{2}+D^{2}\right) /\left(4 G^{2}+D^{2}\right)}$, which yield $\left|\chi_{\mathrm{CCW}}\right| \gg\left|\chi_{\mathrm{CW}}\right|$ for $p=+1$. Alternatively, for $p=-1$, $\left|\chi_{\mathrm{CCW}}\left(\omega_{D}\right)\right|=(|\mu| / \kappa) \sqrt{\left(G^{2}+D^{2}\right) /\left(4 G^{2}+D^{2}\right)}$ and $\left|\chi_{\mathrm{CW}}\left(\omega_{D}\right)\right|$ $=(|\mu| / \kappa) \sqrt{\left(G^{2}+D^{2}\right) / D^{2}}$, thus yielding $\left|\chi_{\mathrm{CCW}}\right| \ll\left|\chi_{\mathrm{CW}}\right|$. As a result, for $p=+1$, the CCW rotational eigenmode has a large motion amplitude, but the $\mathrm{CW}$ rotational eigenmode has an extremely small motion amplitude, and which effect is reversed for $p=-1$.

On the other hand, the $\delta_{\mathrm{CCW}, \mathrm{CW}}^{\mathbf{H}}$ variations with $\omega_{\mathbf{H}}$ are remarkable, owing to its $C$ as well as $p$ dependences. The $C$ dependence of $\delta_{\mathrm{CCW}, \mathrm{HW}}^{\mathbf{H}}$ originates from the $C$ dependence of applied driving forces, ${ }^{26}$ such that $\mathbf{F}_{\mathrm{CCW}}^{\mathbf{H}}=\mu\left(\hat{\mathbf{z}} \times \mathbf{H}_{\mathrm{CCW}}\right)$ $=|\mu| e^{-i C \pi / 2} \mathbf{H}_{\mathrm{CCW}}$ and $\mathbf{F}_{\mathrm{CW}}^{\mathbf{H}}=\mu\left(\hat{\mathbf{z}} \times \mathbf{H}_{\mathrm{CW}}\right)=|\mu| e^{i C \pi / 2} \mathbf{H}_{\mathrm{CW}}$. The $p$ dependence, meanwhile, is due to the already-mentioned asymmetric resonance effect between $\left|\chi_{\mathrm{CCW}}\right|$ and $\left|\chi_{\mathrm{CW}}\right|$. As in the dynamic response of a linear oscillator to a harmonic oscillating force, for $\omega_{\mathbf{H}}<\omega_{D}$, the phase difference $\delta_{\mathrm{CCW}, \mathrm{CW}}^{\mathbf{F}}$ between the $\mathrm{VC}$ position $\mathbf{X}$ and $\mathbf{F}_{\mathrm{CCW}, \mathrm{CW}}^{\mathbf{H}}$ is always zero (i.e., in phase) and independent of $C$ and $p$. However, for the other case, $\omega_{\mathbf{H}}>\omega_{D}, \quad \delta_{\mathrm{CCW}}^{\mathbf{F}}=-\pi(1+p) / 2$, and $\delta_{\mathrm{CW}}^{\mathbf{F}}=-\pi(1-p) / 2$ depend only on $p$. Only for the case of the eigenmode showing resonance, $\delta_{\mathrm{CCW}, \mathrm{CW}}^{\mathbf{F}}$ changes from 0 (in phase) at $\omega_{\mathbf{H}}$ $<\omega_{D}$ to $-\pi$ (out of phase) at $\omega_{\mathbf{H}}>\omega_{D}$ (the second row of Fig. 3). In addition, it is worthwhile noting that such phase change with $\omega_{\mathbf{H}}$ occurs only for the eigenmode showing resonance; it does not occur for the other opposite eigenmode. Thus, the complex changes of $\delta_{\mathrm{CCW}, \mathrm{CW}}^{\mathbf{H}}$ with $\omega_{\mathbf{H}}$, which also depend on $p$ and $C$, can be interpreted simply according to $\delta_{\mathrm{CCW}}^{\mathrm{H}}=\delta_{\mathrm{CCW}}^{\mathrm{F}}+C \pi / 2$ and $\delta_{\mathrm{CW}}^{\mathrm{H}}=\delta_{\mathrm{CW}}^{\mathrm{F}}-C \pi / 2$ (the third row of Fig. 3).

\section{Elliptical shape and orienation of orbital trajectories}

According to the above-mentioned relations of $\left|\chi_{\mathrm{CCW}, \mathrm{CW}}\right|$ and $\delta_{\mathrm{CCW}, \mathrm{CW}}^{\mathbf{H}}$ with $\omega_{\mathbf{H}} / \omega_{D}$ as well as $p$ and $C$, the $\mathrm{CCW}$ and $\mathrm{CW}$ circular-rotational motions can be expressed by the simple mathematical expressions of $\mathbf{X}_{\mathrm{CCW}}=\chi_{\mathrm{CCW}} \mathbf{H}_{\mathrm{CCW}}$ and $\mathbf{X}_{\mathrm{CW}}=\chi_{\mathrm{CW}} \mathbf{H}_{\mathrm{CW}}$, where $\mathbf{H}_{\mathrm{CCW}}=H_{0, \mathrm{CCW}} \exp \left(-i \omega_{\mathbf{H}} t\right) \hat{\mathbf{e}}_{\mathrm{CCW}}$ and $\mathbf{H}_{\mathrm{CW}}=H_{0, \mathrm{CW}} \exp \left(-i \omega_{\mathbf{H}} t\right) \hat{\mathbf{e}}_{\mathrm{CW}}$. Consequently, the superposition of the individual circular eigenmodes thus provides a resultant VC gyrotropic motion driven by an $\mathbf{H}_{\text {lin. }}$. For this concrete verification, the individual orbital trajectories of the $\mathbf{X}_{\mathrm{CCW}}$ and $\mathbf{X}_{\mathrm{CW}}$ and their superposition were also obtained from the analytical calculations, for example, for $\omega_{\mathbf{H}} / \omega_{D}$ $=0.3$ and 2.5 with $H_{0} / H_{A}=0.2$ and for all the cases of $(p, C)$. In Fig. 4, the analytical calculations (solid lines) are in excellent agreements with the simulation results (open circles).

From the relations between the orbital trajectories of the two circular-rotational eigenmodes and their superposition, as shown in Fig. 4, we have found that the degree of elongations of the elliptical trajectories (black-colored lines) for $\omega_{\mathbf{H}} / \omega_{D}=0.3$ and 2.5 is determined by the relative difference between $\left|\mathbf{X}_{\mathrm{CCW}}\right|$ and $\left|\mathbf{X}_{\mathrm{CW}}\right|$ and is independent of $(p, C)$. On the contrary, the elongation axes of the elliptical orbital trajectories corresponding to the motions under an $\mathbf{H}_{\text {lin }}$ are perpendicular to the $\mathbf{H}_{\text {lin }}$ for $\omega_{\mathbf{H}} / \omega_{D}=0.3$ and parallel with the

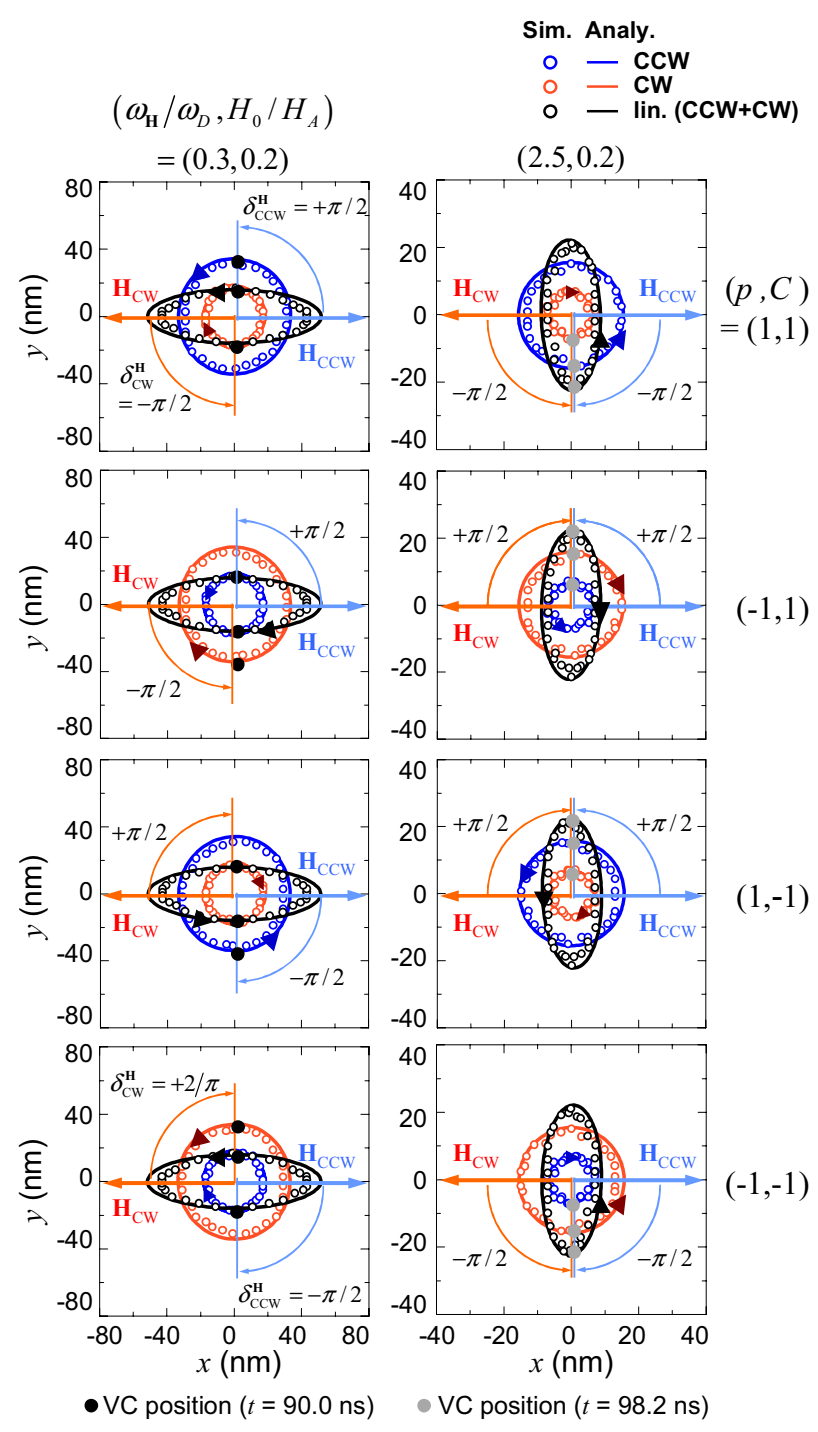

FIG. 4. (Color online) Analytical (solid lines) calculations and simulations (open circles) of the orbital trajectories of the VC motions driven by $\mathbf{H}_{\mathrm{CCW}}$ [blue (dark gray)] and $\mathbf{H}_{\mathrm{CW}}$ [red (light gray)], as well as $\mathbf{H}_{\text {lin }}$ (black) for each case of $(p, C)$. The phase relation between the VC position (closed dots) and the circular field direction (arrows) is illustrated. The arrows on the circular or elliptical orbits indicate their rotation senses.

$\mathbf{H}_{\text {lin }}$ for $\omega_{\mathrm{H}} / \omega_{D}=2.5$. This is associated with the relative phase of $\delta_{\mathrm{CCW}}^{\mathbf{H}}$ and $\delta_{\mathrm{CW}}^{\mathbf{H}}$, i.e., $\left|\delta_{\mathrm{CCW}}^{\mathbf{H}}-\delta_{\mathrm{CW}}^{\mathbf{H}}\right|=\pi$ (out of phase) for $\omega_{\mathbf{H}} / \omega_{D}=0.3<1$ and $\left|\delta_{\mathrm{CCW}}^{\mathbf{H}}-\delta_{\mathrm{CW}}^{\mathbf{H}}\right|=0$ (in phase) for $\omega_{\mathbf{H}} / \omega_{D}=2.5>1$ (see Fig. 3 and Table I). For the case of the out of phase (in phase) between the two eigenmodes, the VC position vectors of the opposite eigenmodes on the applied $\mathbf{H}_{\text {lin }}$ axis are always antiparallel (parallel) to each other, whereas they are always parallel (antiparallel) on the axis perpendicular the $\mathbf{H}_{\text {lin }}$ direction. Thereby, the resulting orbital trajectories of the superposition of the oppositely rotating circular eigenmotions, are ellipse in shape, and their elongation axes are perpendicular (parallel) to the $\mathbf{H}_{\text {lin }}$ direction for $\omega_{\mathbf{H}} / \omega_{D}<1\left(\omega_{\mathbf{H}} / \omega_{D}>1\right)$, which is independent of $(p, C)$.

The rotation sense of the elliptical VC motions is $\mathrm{CCW}$ direction because of $\left|\mathbf{X}_{\mathrm{CCW}}\right|>\left|\mathbf{X}_{\mathrm{CW}}\right|$ for all $p=+1$ cases, 
TABLE I. Numerical estimates of $\delta_{\mathrm{CCW}, \mathrm{CW}}^{\mathbf{F}}\left(\omega_{\mathbf{H}}\right), \delta_{\mathrm{CCW}, \mathrm{CW}}^{\mathbf{H}}\left(\omega_{\mathbf{H}}\right),\left|\chi_{\mathrm{CCW}, \mathrm{CW}}\left(\omega_{\mathbf{H}}\right)\right|, \theta_{\mathbf{G}}$, and $\eta_{\mathbf{G}}$ for the results shown in Fig. 4.

\begin{tabular}{|c|c|c|c|c|c|c|c|c|}
\hline \multirow{2}{*}{$\begin{array}{l}(p, C) \\
\omega_{\mathbf{H}} / \omega_{D}\end{array}$} & \multicolumn{2}{|c|}{$(1,1)$} & \multicolumn{2}{|c|}{$(-1,1)$} & \multicolumn{2}{|c|}{$(1,-1)$} & \multicolumn{2}{|c|}{$(-1,-1)$} \\
\hline & 0.3 & 2.5 & 0.3 & 2.5 & 0.3 & 2.5 & 0.3 & 2.5 \\
\hline$\delta_{\mathrm{CCW}}^{\mathrm{F}}$ & 0 & $-\pi$ & 0 & 0 & 0 & $-\pi$ & 0 & 0 \\
\hline$\delta_{\mathrm{CW}}^{\mathrm{F}}$ & 0 & 0 & 0 & $-\pi$ & 0 & 0 & 0 & $-\pi$ \\
\hline$\delta_{\mathrm{CCW}}^{\mathrm{H}}$ & $\pi / 2$ & $-\pi / 2$ & $\pi / 2$ & $\pi / 2$ & $-\pi / 2$ & $\pi / 2$ & $-\pi / 2$ & $-\pi / 2$ \\
\hline$\delta_{\mathrm{CW}}^{\mathbf{H}}$ & $-\pi / 2$ & $-\pi / 2$ & $-\pi / 2$ & $\pi / 2$ & $\pi / 2$ & $\pi / 2$ & $\pi / 2$ & $-\pi / 2$ \\
\hline$\left|X_{\mathrm{CCW}}\right| /\left|X_{\mathrm{CW}}\right|$ & 1.86 & 2.33 & 0.54 & 0.43 & 1.86 & 2.33 & 0.54 & 0.43 \\
\hline$\eta_{\mathbf{G}}$ & 0.3 & 0.4 & -0.3 & -0.4 & 0.3 & 0.4 & -0.3 & -0.4 \\
\hline$\theta_{\mathbf{G}}$ & $\pi / 2$ & 0 & $\pi / 2$ & 0 & $-\pi / 2$ & 0 & $-\pi / 2$ & 0 \\
\hline
\end{tabular}

whereas thus the rotation sense is $\mathrm{CW}$ direction for all the cases of $p=-1$ because of $\left|\mathbf{X}_{\mathrm{CCW}}\right|<\left|\mathbf{X}_{\mathrm{CW}}\right|$ (see Fig. 3 and Table I). As a result, it is evident that the degree of elongation and its major axis of the elliptical orbital trajectories of those linearly oscillating-field-driven vortex motions shown in Fig. 1(d) are determined by the relations of $\left|\chi_{\mathrm{CCW}, \mathrm{CW}}\right|$ and $\delta_{\mathrm{CCW}, \mathrm{CW}}^{\mathbf{H}}$ with $\omega_{\mathbf{H}}$.

For more quantitative understanding, it is convenient to define the ellipticity $\eta_{\mathbf{G}}$ as the ratio of the length of the major $(a)$ to that of the minor $(b)$ axis, and the rotation $\theta_{\mathbf{G}}$ as the angle of the ellipse's major axis from the $\mathbf{H}_{\text {lin }}$ axis [the $y$ axis in our case, see Fig. 5(a)], as in the Kerr or Faraday ellipticity and rotation in magneto-optics. ${ }^{27}$ The numerical values of $\eta_{\mathbf{G}}$ and $\theta_{\mathbf{G}}$, which are $\eta_{\mathbf{G}}=\left(\left|\mathbf{X}_{\mathrm{CCW}}\right|\right.$ $\left.-\left|\mathbf{X}_{\mathrm{CW}}\right|\right) /\left(\left|\mathbf{X}_{\mathrm{CCW}}\right|+\left|\mathbf{X}_{\mathrm{CW}}\right|\right)=\left(\left|\chi_{\mathrm{CCW}}\right|-\left|\chi_{\mathrm{CW}}\right|\right) /\left(\left|\chi_{\mathrm{CCW}}\right|+\left|\chi_{\mathrm{CW}}\right|\right)$ and $\theta_{\mathbf{G}}=\left(\delta_{\mathrm{CCW}}^{\mathbf{H}}-\delta_{\mathrm{CW}}^{\mathbf{H}}\right) / 2$ by definition, were plotted versus $\omega_{\mathbf{H}} / \omega_{D}$ in Fig. 5(b) for the all cases of $(p, C)$. Note that the analytical calculations (solid lines) of $\eta_{\mathbf{G}}$ and $\theta_{\mathbf{G}}$ are in excellent agreements with the simulation results (open symbols). Owing to the resonance characteristics of either the $\mathrm{CCW}$ or $\mathrm{CW}$ eigenmode for a given $p, \eta_{\mathbf{G}}$ and $\theta_{\mathrm{G}}$ dramatically change across $\omega_{\mathbf{H}} / \omega_{D}=1$ such that $\eta_{\mathbf{G}}=+1$ or -1 (indicating that the orbital trajectory shape is circular) and $\theta_{\mathbf{G}}$ $=+\pi / 4$ or $-\pi / 4$ (Fig. 5). ${ }^{28} \eta_{\mathbf{G}}>0\left(\eta_{\mathbf{G}}<0\right)$ represents the $\mathrm{CCW}(\mathrm{CW})$ rotation of the resultant $\mathrm{VC}$ gyrotropic motion driven by a linearly polarized oscillating field. It is worthwhile noting again that since the relative magnitude of $\left|\chi_{\mathrm{CCW}}\right|$ and $\left|\chi_{\mathrm{CW}}\right|$ is determined by $p$, not by $C$, (i.e., $\left|\chi_{\mathrm{CCW}}\right|>\left|\chi_{\mathrm{CW}}\right|$ for $p=+1$ and $\left|\chi_{\mathrm{CCW}}\right|<\left|\chi_{\mathrm{CW}}\right|$ for $\left.p=-1\right)$, $\eta_{\mathbf{G}}<0$ for $p=-1$ and $\eta_{\mathbf{G}}>0$ for $p=+1$, and by contrast $\theta_{\mathbf{G}}$ depends on both $p$ and $C$ because of their dependences of $\delta_{\mathrm{CCW}, \mathrm{CW}}^{\mathbf{H}}$. The sharp variation of $\theta_{\mathbf{G}}$ from $\pi / 2$ to 0 with increasing $\omega_{\mathbf{H}}$ across $\omega_{D}$ indicates that the major axis of the ellipses changes from the $x$ axis to the $y$ axis. From the calculations of $\delta_{\mathrm{CCW}, \mathrm{CW}}^{\mathrm{H}}$ for each case of $(p, C)$, we can estimate that $\theta_{\mathrm{G}}=+\pi / 2$ or $-\pi / 2$ (the major axis is perpendicular to the $\mathbf{H}_{\text {lin }}$ axis) for $\omega_{\mathbf{H}}<\omega_{D}$, and that $\theta_{\mathbf{G}}=0$ (the major axis is parallel to the $\mathbf{H}_{\text {lin }}$ axis) for $\omega_{\mathbf{H}}>\omega_{D}$, regardless of $p$ and $C$. For all the cases of $(p, C)$, those results are summarized in Table I for the two different cases of $\omega_{\mathbf{H}} / \omega_{D}=0.3$ and 2.5 .

\section{CONCLUSION}

We found that the $\mathrm{CCW}$ and $\mathrm{CW}$ circular-rotational eigenmodes are the elementary eigemmodes existing in the vortex (a)

(b)
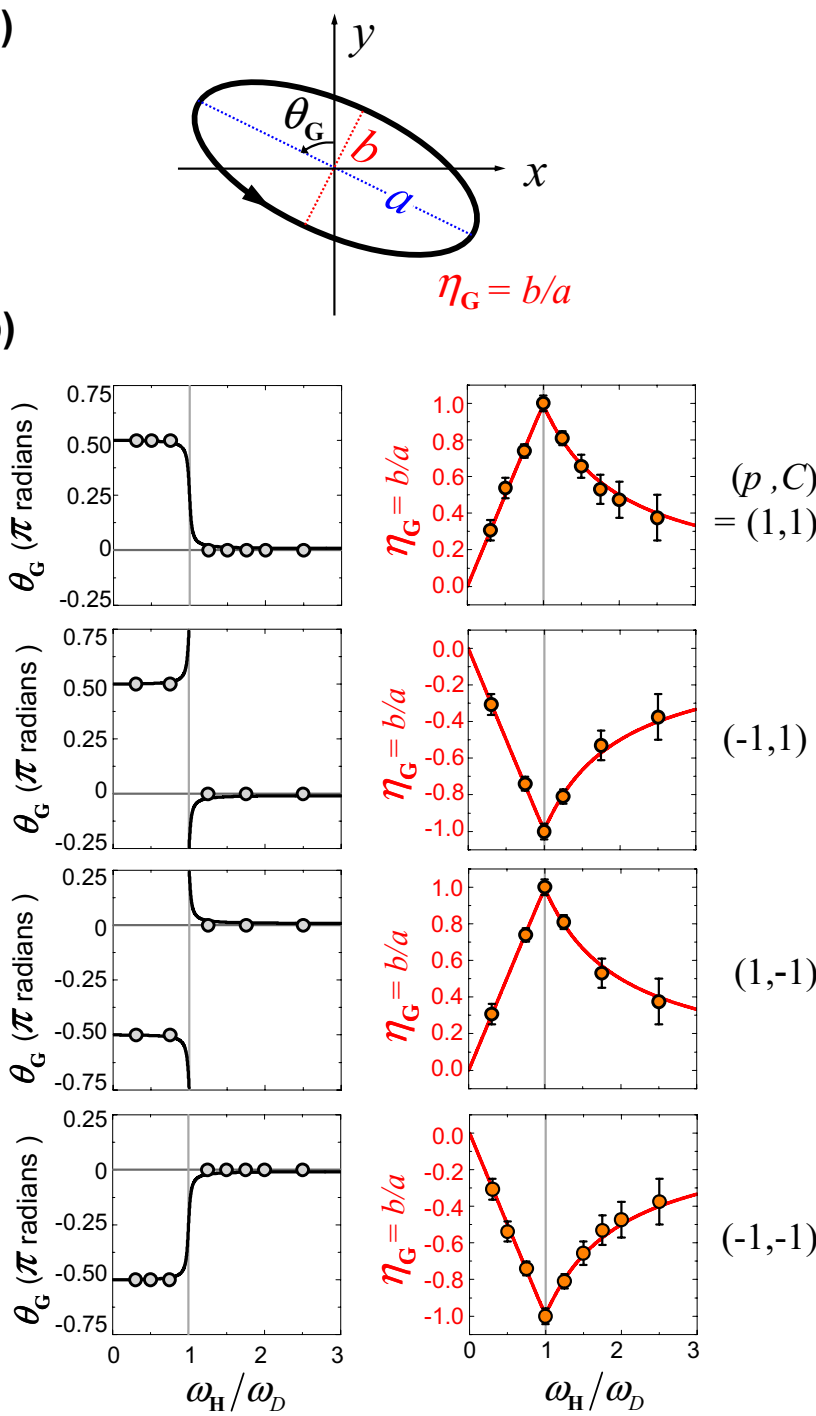

FIG. 5. (Color online) (a) Illustration of the definitions of $\theta_{\mathbf{G}}$ and $\eta_{\mathbf{G}}$ described in the text. (b) Numerical estimates of $\theta_{\mathbf{G}}$ and $\eta_{\mathbf{G}}$ from the micromagnetic simulations (symbols) and numerical calculations of the analytical equations (solid lines) for all cases of $(p, C)$, as noted. The simulation results correspond to the cases shown in the first column in Fig. 1(d). 
gyrotropic motions in circular nanodots and that the two eigenmodes' resonant excitations are largely asymmetric, according to the vortex polarization. The relative magnitudes in the orbital-radius amplitude and phase between the two circular eigenmodes determine the elongation and orientation, respectively, of the orbital trajectories of the vortex core motions driven by a linearly oscillating field. These results provide information on how the orbital-radius amplitude and phase of a vortex core motion vary with the polarization and chirality of the given vortex state as well as the field frequency, resulting in overall linear-regime steady-state vortex gyrotropic motions driven by any polarized oscillating fields. Due to the distinctly different asymmetric resonance effect between the $\mathrm{CCW}$ and $\mathrm{CW}$ circular motions on resonance, the $\mathrm{CCW}$ and $\mathrm{CW}$ circular fields with the resonance frequency can be used to selectively switch upward and downward oriented vortex cores, respectively, as well as to selec- tively excite the large-amplitude $\mathrm{CCW}$ and $\mathrm{CW}$ gyrotropic motions of the upward and downward vortex cores, respectively. From a technological point of view, these allow for selective, reliable switching of the magnetization of vortex cores with low power consumption and the indirect detection of the $\mathrm{VC}$ orientation by monitoring the large shift of the vortex core position or the largely asymmetric in-plane $\mathbf{M}$ orientations through directly measuring induced voltage or tunneling magnetoresistance and giant magnetoresistance contrast with pinned reference spin configurations in the other ferromagnetic layers.

\section{ACKNOWLEDGMENT}

This work was supported by Creative Research Initiatives (Research Center for Spin Dynamics and Spin-Wave Devices) of MOST/KOSEF.
*Corresponding author; sangkoog@snu.ac.kr

${ }^{1}$ T. Shinjo, T. Okuno, R. Hassdorf, K. Shigeto, and T. Ono, Science 289, 930 (2000).

${ }^{2}$ A. Wachowiak, J. Wiebe, M. Bode, O. Pietzsch, M. Morgenstern, and R. Wiesendanger, Science 298, 577 (2002).

${ }^{3}$ S.-K. Kim, J. B. Kortright, and S.-C. Shin, Appl. Phys. Lett. 78, 2742 (2001); S.-K. Kim, K.-S. Lee, B.-W. Kang, K.-J. Lee, and J. B. Kortright, ibid. 86, 052504 (2005).

${ }^{4}$ S. B. Choe, Y. Acremann, A. Scholl, A. Bauer, A. Doran, J. Stohr, and H. A. Padmore, Science 304, 420 (2004).

${ }^{5}$ K. Y. Guslienko, B. A. Ivanov, V. Novosad, Y. Otani, H. Shima, and K. Fukamichi, J. Appl. Phys. 91, 8037 (2002); K. Y. Guslienko, X. F. Han, D. J. Keavney, R. Divan, and S. D. Bader, Phys. Rev. Lett. 96, 067205 (2006).

${ }^{6}$ J. P. Park, P. Eames, D. M. Engebretson, J. Berezovsky, and P. A. Crowell, Phys. Rev. B 67, 020403(R) (2003).

${ }^{7}$ K.-S. Lee, K. Y. Guslienko, J.-Y. Lee, and S.-K. Kim, Phys. Rev. B 76, 174410 (2007).

${ }^{8}$ K.-S. Lee and S.-K. Kim, Appl. Phys. Lett. 91, 132511 (2007).

${ }^{9}$ K. S. Buchanan, M. Grimsditch, F. Y. Fradin, S. D. Bader, and V. Novosad, Phys. Rev. Lett. 99, 267201 (2007).

${ }^{10}$ S. Kasai, Y. Nakatani, K. Kobayshi, H. Kohno, and T. Ono, Phys. Rev. Lett. 97, 107204 (2006).

${ }^{11}$ B. Kruger, A. Drews, M. Bolte, U. Merkt, D. Pfannkuche, and G. Meier, Phys. Rev. B 76, 224426 (2007)

${ }^{12}$ K. Y. Guslienko, Appl. Phys. Lett. 89, 022510 (2006).

${ }^{13}$ S. Choi, K.-S. Lee, K. Y. Guslienko, and S.-K. Kim, Phys. Rev. Lett. 98, 087205 (2007)

${ }^{14}$ B. Van Waeyenberge, A. Puzic, H. Stoll, K. W. Chou, T. Tyliszczak, R. Hertel, M. Fähnle, H. Brückl, K. Rott, G. Reiss, I. Neudecker, D. Weiss, C. H. Back, and G. Schütz, Nature (London) 444, 461 (2006); Q. F. Xiao, J. Rudge, B. C. Choi, Y. K. Hong, and G. Donohoe, Appl. Phys. Lett. 89, 262507 (2006); R. Hertel, S. Gliga, M. Fähnle, and C. M. Schneider, Phys. Rev. Lett. 98, 117201 (2007); K. Y. Guslienko, K.-S. Lee, and S.-K. Kim, ibid. 100, 027203 (2008)

${ }^{15}$ S.-K. Kim, K.-S. Lee, Y.-S. Yu, and Y.-S. Choi, Appl. Phys. Lett. 92, 022509 (2008).

${ }^{16}$ K. Yamada, S. Kasai, Y. Nakatani, K. Kobayashi, H. Kohno, A.
Thiaville, and T. Ono, Nat. Mater. 6, 269 (2007); R. P. Cowburn, ibid. 6, 255 (2007); J. Thomas, Nat. Nanotechnol. 2, 206 (2007).

${ }^{17}$ S.-K. Kim, Y.-S. Choi, K.-S. Lee, K. Y. Guslienko, and D.-E. Jeong, Appl. Phys. Lett. 91, 082506 (2007).

${ }^{18}$ Denis D. Sheka, Yuri Gaididei, and Franz G. Mertens, Appl. Phys. Lett. 91, 082509 (2007)

${ }^{19}$ See http://math.nist.gov/oommf

${ }^{20}$ L. D. Landau and E. M. Lifshitz, Phys. Z. Sowjetunion 8, 153 (1935); T. L. Gilbert, Phys. Rev. 100, 1243 (1955).

${ }^{21}$ A. A. Thiele, Phys. Rev. Lett. 30, 230 (1973); D. L. Huber, Phys. Rev. B 26, 3758 (1982).

${ }^{22}$ K. Y. Guslienko, V. Novosad, Y. Otani, H. Shima, and K. Fukamichi, Phys. Rev. B 65, 024414 (2002).

${ }^{23}$ Note that we used $H_{0} / H_{A}=0.01$ to avoid VC escape from the dot (Ref. 7)

${ }^{24}$ See EPAPS Document No. E-PRBMDO-77-084817 for two movie files. Supplementary Movie 1: animation on the temporal evolution of the spatial configuration of the local magnetizations and of the vortex-core motion for the case shown in Figs. 1(b) and 1(c). Supplementary Movie 2: animation on the temporal evolution of the individual $\mathrm{CCW}$ and $\mathrm{CW}$ circular rotational eigenmodes and of the corresponding circular eigenbasis of the pure circularly rotating fields, $\mathbf{H}_{\mathrm{CCW}}$ and $\mathbf{H}_{\mathrm{CW}}$, and with their superposition. For more information on EPAPS, see http:// www.aip.org/pubservs/epaps.html.

${ }^{25}$ Note that such an eigenbasis can be chosen according to the symmetry of $W(\mathbf{X})$. For an elliptical dot, an elliptical-rotatingfield basis can be used as the eigenbasis.

${ }^{26}$ For the case of oscillating spin-polarized currents, there is no $C$ dependence of applied driving forces. Consequently, the phase $\delta_{\mathrm{CCW}, \mathrm{CW}}^{\mathrm{I}}$, does not depend on $C$.

${ }^{27}$ K.-S. Lee, D.-E. Jeong, S.-K. Kim, and J. B. Kortright, J. Appl. Phys. 97, 083519 (2005); D.-E. Jeong, K.-S. Lee, and S.-K. Kim, J. Korean Phys. Soc. 46, 1180 (2005).

${ }^{28}$ Here, we emphasize only the linear regime for relatively low values of $H_{0} / H_{A}$. As far as the linear regime is considered, the values of $\eta_{\mathbf{G}}$ and $\theta_{\mathbf{G}}$ do not change with $H_{0} / H_{A}$. 